\title{
Attitudes Towards Gambling and Gambling Reform in Australia
}




\section{Authors and Acknowledgements}

\section{Phillip Donaldson}

Matthew Rockloff

Casey-Marie Sorenson

Matthew Browne

\section{En Li}

Institute for Health and Social Science Research

- Australian Health and Social Science study (AHSS)

- CQU Population Research Laboratory (PRL) 


\section{Attitudes Towards Gambling}

- The need to assess public attitudes towards gambling

- Original UK context of the ATGS

- The need to evaluate the ATGS in an Australian context

- Differences in policy and population

- What does the ATGS tell us about attitudes among Australians?

- Validity of the scale

- Utility of the scale 


\section{Design and Method}

- Partial replication of original UK study

- Orford, Griffiths, Wardle, Sproston \& Erens (2009)

- 1794 Australian adult AHSS members (52.8\% female) recruited by CATI

- Survey items:

- ATGS

- Attitudes towards Legislative Change

- Experiences with Gambling (CPGI)

- Demographics 


\section{Attitudes Towards Gambling Scale}

- 14 items

- "Gambling should be discouraged"

- "Gambling is a harmless form of entertainment"

- Items selected to be independent of specific forms of gambling, cohorts, and policy issues

- Correlations between $0.5-0.7$

- Range 14 - 70

- Higher scores associated with more positive attitudes towards gambling 


\section{Attitudes Towards Legislative Change}

- Attitudes Towards The Poker Machine Harm Reduction (\$1 Bets and Other Measures) Bill 2012

- 6 items

- Range 6-24 (higher scores in favour of reforms)

- Attitudes Towards The Interactive Gambling and Broadcasting Amendment (Online Transactions and Other Measures) Bill 2011

- 15 items

- Range 15 - 60 (higher scores in favour of reforms)

- Experiences with Gambling

- Questions adapted from the Canadian Problem Gambling Index (PGSI)

- Frequency, type of gambling, risk indicators 


\section{Validity of ATGS}

- Internal Reliability

- Cronbach's $\alpha=0.88$ (14 items)

- Item correlations $0.2-0.6$ (all sig at $<.001$ )

- Principle Components Analysis

- Produced two factors

- First factor accounted for $39.5 \%$ of variance

- Second factor accounted for $9.05 \%$ of variance

- Factors appear to reflect method with positively worded items loading onto first factor and negatively worded items loading onto second factor

- Conclusion - ATGS constitutes a satisfactory attitude scale for use with an Australian population 


\section{Attitudes Among Australian Population}

- Mean ATGS score $=32.5(S D=8.5)$

- Attitudes generally moderately negative (mid-point $=42)$

- UK population $M=35.25, S D=8.52$

- Mean Attitudes Towards Harm Reduction $18.4(S D=3.5)$

- Moderately positive (Range $6-24$, mid-point $=15$ )

- ATGS accounts for $22.1 \%$ of variance $(\mathrm{R} 2=.22, F(1,1792)=508.84, p$ $<.001, \beta=-.47)$

- Mean Attitudes Towards Reforms = $49.7(S D=6.8)$

- Moderately positive (Range $15-60$, mid-point $=37.5$ )

- ATGS accounts for $25.3 \%$ of variance $(\mathrm{R} 2=.25, F(1,890)=303.29, p<$ $.001, \beta=-.51)$ 


\section{Gambling Experiences and the ATGS}

Variable

Frequency of Gambling (by type)

Lottery Tickets

Instant Scratch-it Tickets

Horse or Dog Racing

Sports Tipping

Cards, darts, pool etc.

Sports through a bookie

Time since most recent gambling

Gambling Risk Factors

Age of first gambling experience

Age of first gambling with peers or immediate family

${ }^{*} p<.05, * * p<.001$
$B$ Value

$.137^{* *}$

$.112^{* *}$

$.129 * *$

$.054 *$

.053*

.059*

$.418^{* *}$

$-.063 *$

$-.143^{* *}$

$-.129 * *$ 


\section{Demographics and the ATGS}

\begin{tabular}{lll} 
Variable & FValue* & Direction (more positive) \\
\hline Preferred form of gambling & 10.94 & Pokies and sports betting \\
Sex (M/F) & 6.36 & Male \\
Highest Level of Education & 1.48 & No response \\
Current Employment Status & 1.86 & Unemployed, home duties, \\
Personal Income & & no response \\
State of Residence & 2.5 & 70,000 - 150,000 p.a. \\
\hline
\end{tabular}

No Significant effects of:

-Age, Marital status, Country of birth, Aboriginal or Torres Strait Islander status, Total family income 


\section{Summary and Conclusions}

- The Attitudes Towards Gambling Scale

- valid measure of attitudes towards gambling among an Australian population

- useful predictor of attitudes towards other gambling related issues

- should be supplemented by other, modality specific measures

- Comparisons between UK and Australian populations

- Australian population more homogenous than UK

- Social class barriers less influential

- Attitudes among the Australian population

- Generally negative towards gambling, positive towards reform

- More positive among regular poker machine players, unemployed, frequent and recent gamblers, those who started young and males (however, still fall on the negative end of the scale) 